\title{
Improving Student Engagement and Performance in Computing Final Year Projects
}

\begin{abstract}
There has been a seismic shift in the UK higher education landscape during the last decade. This has been driven by the formation of the Office for Students (OfS) and the introduction of the Teaching Excellence Framework (TEF), where the emphasis has been on programmes offering students higher value when it comes to employability, retention and overall student experience. One of the critical challenges that impacts student experience is being able to enhance student engagement within a learning environment. Final year individual projects, which are generally unstructured in nature, is a significant contributor to programmes of study, yet remains an area where this problem is exacerbated. In an attempt to address this issue, our earlier work lays the foundation for a teaching \& learning framework covering computing final year projects. In this paper, we present an extension to the framework and its implementation in 2016/17 following its first trial run within a Computer Science department at a UK university in 2015/16. We discuss the two implementations in practice and provide operational guidance. A large-scale longitudinal empirical study considering the performance of 625 final year undergraduate students over a period of five years is presented to ascertain the effectiveness of the framework. The study finds a consistent and significant positive impact on both student performance and engagement as a result of the original framework and further gains from the enhancements.
\end{abstract}

Index Terms-Teaching/learning strategies, student engagement, computing projects, higher education

\section{INTRODUCTION}

Over the years there have been significant changes in teaching and learning approaches used in Higher Education (HE). In particular, student engagement is being considered the most important aspect and has become a worrying concern among the HE institutions. There is a strong emphasis on degree programmes having to offer higher value [9] regarding aspects such as - engagement, retention, employability and overall student experience [15] as emphasized by regulatory and government policies. A critical factor that influences student experience is the ability to engage students effectively, as poor student engagement can negatively impact student experience. Hence, institutions have been moving away from traditional methods to motivate and engage students. The focus is primarily on the adoption of different techniques to motivate and engage students [13]. A challenge in developing such techniques is being able to create educational experiences that can trigger and keep the interest of students. A way to achieve this is by increasing the levels of motivation and enjoyment within the student's learning activities and milestones [21], [25].

Learning experiences can be enhanced by active learning techniques that are widely accepted more impactful compared to the traditional techniques of enhancing student engagement. This is because active learning methods can enhance learning experiences and provide improved motivation using rewarded accomplishments [8]. In order to enhance student engagement, the learning environment and individual learning must be regarded as one due to cross-cutting influences [5]. One approach for Final Year Project (FYP) modules is to support a learning environment which fosters collaboration and communication between supervisors and students. This develops a sense of co-operation and belonging within the student, positively impacting student engagement [25]. In relation to this, the work in this paper proposes an extension to an existing framework designed to provide complete support for modules covering final year projects [2]. The main focus of this framework is to improve student engagement and overall student experience. The development methodology of this framework and the extensions proposed in this paper uses the Technical Action Research Model [6]. At the initial formulation we incorporated Pickerings Taxonomy [20] and Brookfield's Critical Lenses [4] for development of the framework. The extension is the iterative improvements based on the first implementation of the framework and feedback from all the stakeholders as per the action research model. To the best of our knowledge, our framework is the first formalized published approach for computing final year projects.

This paper makes the following contributions:

- Extend our existing framework [2] by introducing a final project proposal milestone and vetting, which enables early formative assessment, feedback and assured engagement. To the best of our knowledge, we are the first to propose such a vetting process for FYPs.

- Provide practical implementation considerations for the framework, which involves selection process of assessors, training workshops for supervisors and students and the marking process.

- An extensive longitudinal empirical validation over the period of 5 years considering the performance of 625 undergraduate students in the subject domain of Computer Science in their FYPs to assess the impact and the effectiveness of the framework.

The rest of this paper is structured as follows, Section II provides background of the related work, Section III discusses the development approach, while Section IV describes the developed framework and its implementations. Section V presents experimental validation and identifies threats to the validity of the experimental results. Finally, Section VI presents our 
conclusions and identifies future extensions.

\section{RELATED WORK}

Final Year Project (FYP) is a central assessment component of an undergraduate programme, as it expects learners to undertake a self-directd learning journey that empowers them to strengthen their problem-solving and research skills [19]. Healey et al. [11] argues that all undergraduate projects must be part of every undergraduate course, irrespective of the discipline of study as it provides students with opportunity to develop skills and knowledge outside the taught environment. The project should be in line with the interests of both student and the supervisor as this a significant impact on the motivation level of the learner and their engagement with the project which will be reflected in the overall student experience. However, good student experience may not necessarily reflect good performance as the project is a long term commitment towards a single goal, typically year long in the UK institutions and keeping students engaged and motivated along with decent performance can be challenging in such long period. These factors along with timely guidance and mentoring play an important role [5] and influences the student's motivation to learn and work efficiently towards achieving their goals within their programme of study [17]. Hence, the focus should primarily be based on the adoption of different techniques that not only motivate and engage students [13] but also create educational experiences that can trigger and keep the interest of the students. Shay [24] presented an extensive study on academic professional judgment for FYPs and finds that substantial structuring and clarity should support the process. We have included sufficient structuring around the requirements and assessment of our framework to ensure that while allowing for academic judgment the criteria and its application is open and transparent.

FYPs belong to the category of unstructured learning as the learning process is not predefined and not based on a planned delivery or guidance from specific textbooks. The learning process involves individual effort to conduct research that blends with the interest and capabilities of the students. Consequently, most of the learning process is based on independent research, blended learning, collaborations and feedback [26]. Use of technology in teaching and learning is regarded as a vital approach to support these. One of the reasons is that technology can be considered a necessity as opposed to a requirement as it offers more than just being a repository for the content delivery rather, technology can enable interaction, feedback and engagement that enhances the student's learning experience [22]. In the past various attempts have been made to enhance student experience and reinforce student competence in Final Year Projects by making it more engaging and interactive. Our proposed framework stands on the foundations backed by technology which provides a platform for engagement, collaboration and monitoring assessments.

An extensive study by Healey et al. [11] looked into $70+$ case studies of FYP implementations, which showed that various attempts have been made by UK institutions to make FYPs more engaging for the students by introducing different assessment methods, empowering students with decision making, guided supervision, skills development, collaborative working, engaging community groups and the employers which are identified as the key attributes that can play vital role towards enhancing student experience. However, these approaches lacked a framework that could provide a mechanism to embed these on a single platform. This has been primarily attempted by introducing what we have proposed and adopted in our framework, i.e use of technologies in teaching and learning, that has been highlighted in some of the following examples. For example, an attempt by the University of Portsmouth, UK was the Technology Assisted Project Supervision TAPaS) [16]. This approach made use of mixed technologies and tools comprising of e-logs, twitter, web forums and Wiki to establish a framework that could be used to improve communication and collaboration in the supervision of FYPs. The results from this study found that the use of technology had a positive impact on the student engagement and supervision process. However, the study also suggested that the use of multiple formal and informal communication and collaboration tools was not welcomed by most students and supervisors who showed concerns with regard to plagiarism for using technologies such as twitter for communication that involved important piece of assessment in a degree course. This approached also showed that it was fairly difficult to accumulate all the relevant information from different tools and resources for both students and the supervisors.

Another example of technology adoption was InterDisciplinary Inquiry-Based Learning (IDIBL), which was an online collaborative framework implemented by University of Bolton, UK focused on action research. This was more of a communication platform where peers could seek support and guidance from each other and work towards a required objective. Therefore it will be unfair to regard this approach as a viable mechanism for monitoring and enhancing student performances.

Similar attempts have also been made by other institutions internationally such as use of Moodle [14] and in-house developed online system, Online Project Evaluation and Supervision System (oPENs) [12]. In the first attempt, Moodle could not serve the purpose of enhancing student experience or engagement as it was more of a content repository than an interactive and a monitoring tool. On the other hand, oPENS was a user developed online system that was able to provide some functionality enabling project evaluation and monitoring but limited in providing a mechanism of control, feedback and interaction with the students. The attempts mentioned have been towards implementing a tools to monitor project progress rather than facilitating a single teaching and learning framework that can be implemented effectively to enhance student engagement, motivation and performance. We take lessons learned from each of these approaches and hence propose a framework that will address the above more efficiently and 
effectively.

Our enhanced framework addresses these key issues and provides a practical implementation that will enhance the overall student engagement and experience in the final year projects by not only streamlining the overall supervision process but also provide the necessary communication, collaboration, assessment, control and monitoring under a single platform. In order to achieve these we have diversified assessment within our FYP framework including series of formative assessments in vetting the project proposal, even assessing and rewarding continued engagement, and, include project demonstration as a key milestone. Additionally, The study also highlights the importance of end-of-year shows and presentations to motivate and attract interest and the ownership of projects.

Diversification in assessment is important, as Bloxam et al. [3] suggests that diversifying the assessment not only judges the students but also provides them an opportunity to put forward their factual and conceptual knowledge of their chosen topic and this can be enhanced by allowing them to showcase their work as we propose in our framework.

\section{Methodology}

The development methodology used for the enhanced framework uses Technical Action Research Model. The goal of the model is to assess an existing situation and make it more efficient and effective [6]. The model has the following stages - Initial Reflection, Planning, Action and Observation.

\section{A. Initial Reflection}

For the initial stage, Pickering [20] questions supplemented by Brookfields lenses [4] were used to reflect on the issues with the current FYP module. Brookfield's lenses is a wellestablished framework for identifying problems associated with various aspects of teaching and learning, while Pickering questions are more oriented towards the inclusion of technology into the curriculum. A summary of findings from this initial stage echoed similar other studies [10], and highlighted the following issues:

- Limited instructional scaffolding to aid the continued learning process throughout the year.

- Students lacked project management skills and found it challenging to manage an extensive year-long individual assessment (project).

- Students struggled with independent research and expected unstructured learning.

- Lack of organized feedback during the year-long engagement.

- No consistent mechanism for measuring levels of continuous engagement.

- Difficult to measure progress until the very end.

\section{B. Planning}

Effective pedagogy is dependent on learning being systematically developed [27], which in turn leads to instructional scaffolding [23]. Taking this into consideration, this stage involved making modifications to the module specification, assessment structure and the module study guide. In addition to this, the impact on staff and logistics were also considered. Based on this, the project module was structured to implement the framework and its components depicted in Figure 1 and described in Section IV. These modifications were carried out to enhance the teaching quality and the learning process for the project module, with the ultimate goal of getting students to perform better on the final year projects.

\section{Action}

In this stage, we implement the updates within the context of the institutional change management process and training workshops for the supervisors and their students. The topic of student project sites, which enables students to manage and showcase their projects professionally is also covered as a topic during the workshops.

\section{Observation}

During the this phase, quantitative and qualitative statistics were produced to identify the impact of the (original) framework for its first implementation in 2015/16. The quantitative data was in the form of student performance and the qualitative data was in the form of module evaluation done by students and feedback from supervisors.

Evaluation and reflection of the data led to further (action) enhancements to the framework for the second implementation (2016/17) resulting in further positive improvements. In particular, the final proposal vetting process was introduced into the enhanced framework during the second iteration to successfully improve both engagement and the pass rate. Iterative improvements are an integral part of the Action Research model.

\section{FRAMEWORK}

FYP presents the students with the opportunity to work on an substantial problem in the computing domain. The project affords the opportunity for students to apply technical skills acquired during their course. The project is also an opportunity for students to demonstrate their problem resolution and independent research skills, which includes a substantial amount of computing/scientific/engineering content. The projects encompass components such as software / application development, mathematical modeling, empirical research, engineering or scientific methodology, commercialization, and, legal, social and ethical considerations.

The goal of the enhanced framework (Figure 1) is to improve student engagement and performance during a year-long individual project that undergraduate final year Computing students are expected to complete for their degree award. In an attempt to improve student engagement and performance from previous years, the framework incorporates progress monitoring checkpoints and continuous feedback throughout the lifespan of the project. This feedback is made possible through the integration of a sequence of assessments covering both formative and summative types. 
TABLE I

ASSESSMENT BREAKDOWN

\begin{tabular}{|c|c|c|c|}
\hline \multicolumn{3}{|c|}{ Supporting Project Material $-\mathbf{2 5 \%}$} & Main Project Report - 75\% \\
\hline
\end{tabular}

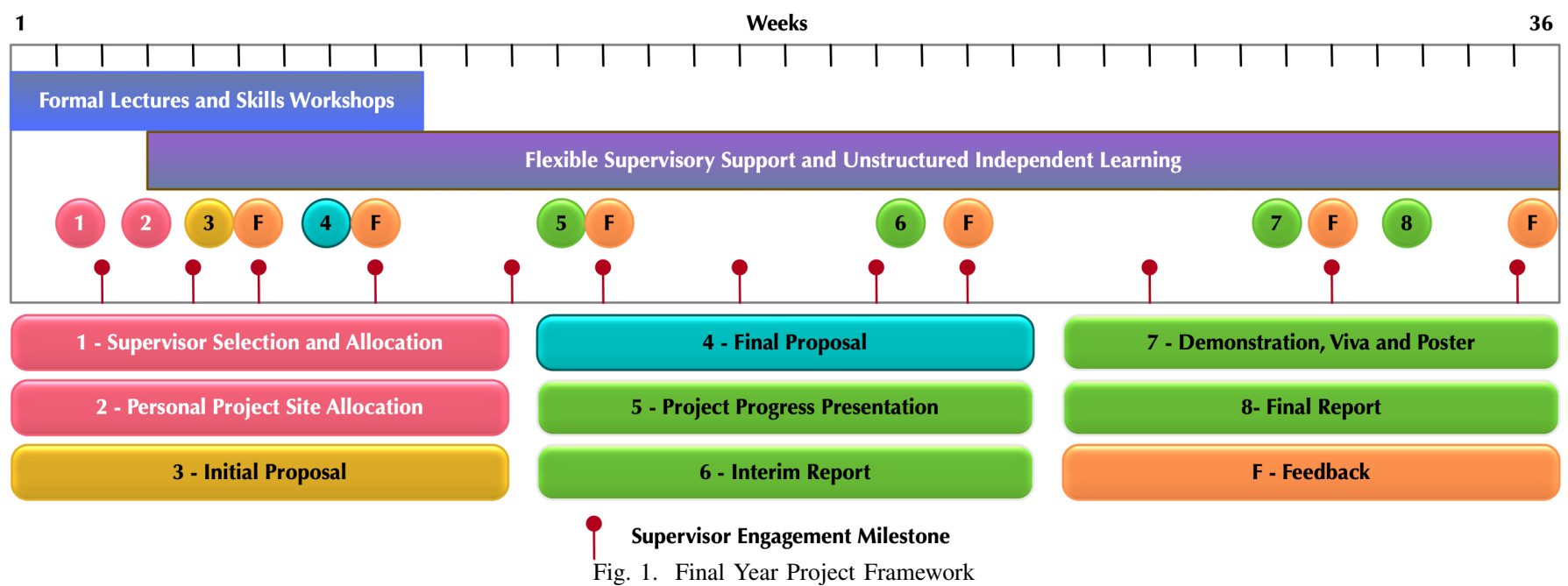

The framework is structured around the module construct that is used to deliver and manage the module. It takes into consideration progression, epistemology, benchmarking and the overall curriculum . Specifically, we focused on parts of the curriculum that needed refreshing and project tasks that students traditionally struggled with, e.g. writing literature reviews, project planning and identifying and applying appropriate methodology and scientific or engineering rigor. The framework also considered programme \& institutional context and benchmarking to the British Computer Society (BCS) and Quality Assurance Agency for Higher Education (QAA) standards. These aspects were primarily handled by a series of Formal Lecture and Skills Workshops (Figure 1 blue). The assessment breakdown for our implementations of the framework is given in Table I.

In addition to the formal lectures and workshops, the framework (Figure 1) includes a range of summative (yellow and teal : 3-4) and formative assessments (green: 5-8) as milestones that assist students in working on their projects during the project life-cycle. This is a significant change in comparison to the previous structure of the project module which had a single summative assessment (final project report), where the students were expected to submit at the end of the academic year.

The purpose of the milestones is to ensure that students are encouraged and directed to cover components that are required for a good project and provides regular feedback (Figure 1 - orange : F). The final year project framework has been designed in this way to ensure that it is comprehensive and can provide students with scaffolding support. The sessions with the diagnostic phases and the flexibility within the framework can accommodate students with a wide range of abilities.
The level of support (from the support staff and supervisors) can be adapted to satisfy the specific needs of every student, which is based on research, thinking, problem-solving, time management or subject-specific practical skills. This paper reports on the findings of two deployments of the framework, the first deployment was for the original framework [2] during the academic year 2015/16 (Implementation 1), and, the second deployment was with enhancements (Implementation 2) in 2016/17. The next four sub-sections details the support structures, milestones and assessment built into the final year project module reflecting the enhanced framework and some practical implementation guidance.

\section{A. Original Framework Milestones - Implementation 1 (2015/16)}

1) Supervisor Selection and Allocation: The first step of the project process requires students to select a supervisor, which must be done within a two-week period (Figure 1 - pink : 1). The allocated supervisor will supervise the student for the entire duration of the project. The sole objective of supervision is to equip students with academic expertise based on the topic being investigated for the project. The supervisor's role is that of a mentor who guides the students through various steps of the project.

2) Engagement with Supervisor: Students are sometimes daunted by managing and working on a year-long piece of assessment. Therefore the framework encourages the supervisors to have a minimum of 12 meetings with the student. These meetings are classed as feedback milestones. (Figure 1 - red dot). These sessions allow supervisors to measure the student's progress and facilitate instructional scaffolding supporting the student's abilities. These engagement periods are crucial in 
maintaining student expectations and helping students engage with the project, which in turn improves retention.

3) Initial Project Proposal: Students are required to produce their proposals (Figure 1 - yellow : 3) after they have had an initial meeting with their supervisor. This proposal outlines the project aims and the justification for choices and the skills and resources required to complete the project.

4) Student Project Site: One of the significant features employed within the framework is the creation of individual student SharePoint project sites (Figure 1 - pink : 2). This is motivated by the advantages of utilising technology to enhance and support learning. The project sites are used for several features such as a workspace for projects, submission of project documents, project management, sharing documents and drafts with supervisors, an integrated notebook for logs. The project site also provides a planning tool that enables students to implement project planning \& management skills. The technology supports continuous formative and summative assessment. We used TAM [7] to validate this technology inclusion and found that users reported high perceived usefulness and perceived ease of use.

5) Project Progress Review Presentation: During week 12 of the project, students are required to participate in a project progress review presentation (Figure 1 - green : 5). The purpose of this review is to assess the student's project progress, literature review findings, proposed implementation design and a project plan. The review panel comprises of the student's supervisor and an independent assessor who decide if satisfactory progress was made and provide feedback to the student.

6) Project Reports: During the course of the project, students are required to write two reports, namely an interim report and a final report. The Interim Report (Figure 1 - green :6) should incorporate the literature review, the design of the solution and a project plan. This report is submitted after the progress review presentation, which gives the students an opportunity to reflect on the presentation feedback and address any suggestions in the interim report. The Final Report (Figure 1 - green : 8) is documentation that reflects the entire project work, which is due at the end of the project. The report includes an abstract, problem definition, proposed solution description and state a conclusion regarding the success of the solution. In addition to this, the main body of the report would usually cover traditional sections such as requirements analysis, design, implementation, testing, results, validation, evaluation/discussion, conclusion and references.

7) Demonstration \& Poster Assessment Event: A significant component of the project assessment requires the students to participate in the demonstration and poster event (Figure 1 - green : 7). During this period, students would have completed their implementation and be able to demonstrate their project solution. This is done in the form of students exhibiting their project work by presenting their poster and giving a demonstration. This event is a motivation driver as it gives students a sense of ownership and opportunity to demonstrate their projects openly to the external audiences.
This is a crucial part of the project assessment, which also contributes to the summative marks. Students who fail to attend the demonstration event are awarded an automatic fail for the project. This highlights the significance of the practical demonstrations which are essential for the Computer Science discipline.

8) Viva Presentation: The viva assessment (Figure 1 - green $: 7)$ requires the students to create a presentation to explain their project work. The goal of the viva is to concentrate on the practical aspects of the project, where students are expected to answer questions about the design, implementation and limitations of their projects. The viva also provides assessors with an opportunity to interact with students and ask them to provide further clarification on aspects of their project report or demonstration.

\section{B. Enhanced Framework Milestones - Implementation 2 (2016/17)}

For Implementation 1, we did a quantitative and qualitative analysis of the performance of the framework. We found that a significant portion of the students who failed the module was not only because they produced poor quality work but also because their projects were either infeasible or did not meet the benchmarking standards. In light of the findings, we introduced an enhancement to the framework before its second deployment in 2016/17 resulting in improvement of both student and engagement and student performance (Section V-A).

In additions to the milestones outlined in Implementation 1, the main enhancement for Implementation 2 was the inclusion of a final proposal milestone and its vetting process to ensure that students do not embark on projects that are infeasible or those that fall short of the required standards.

1) Final Proposal and Vetting: The final proposal (Figure 1 - teal : 4) and its vetting process is an important milestone and builds on the initial proposal. In order for a student to continue with their project (i.e. not being withdrawn from the module), their final proposal needs to be approved by their supervisor and the second marker, who is an independent assessor for the project. The second marker does not have any interaction with the student throughout the project process, i.e. the student cannot get supervisory support from the second marker.

Students are given two attempts at producing the final proposal for approval. If the student's first proposal does not satisfy the assessment criteria then they are afforded a week to make revisions to their proposals. If at the second attempt the student's proposal still does not meet the criteria, they are withdrawn from the module. The introduction of this milestone is to ensure that students do not continue working on a project that is highly likely to lead to a failed project. Figure 2 outlines the flowchart for the Final Proposal submission and withdrawal process.

The introduction of this final proposal vetting process had the following impact:

- ensured project ideas met the guidelines and requirements laid out by the institutional and national standards. 


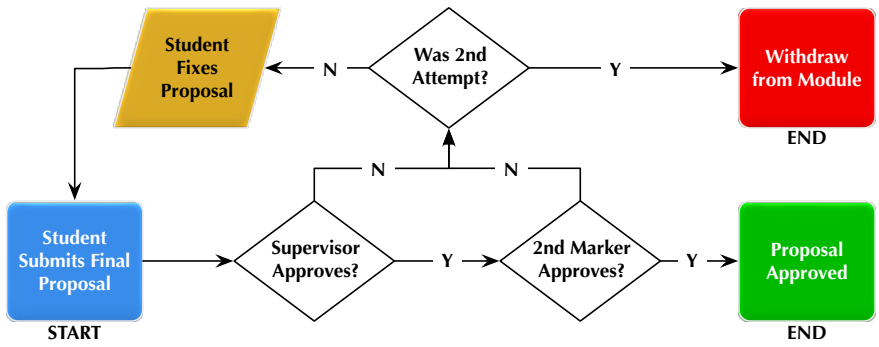

Fig. 2. Final Proposal Vetting Process

- ensured a realistic project scoping and planning which would maximize the possibility of completion.

- ensured required skills and resources were identified along with a plan for their acquisition.

At first glance, the inclusion of the proposal vetting and withdrawal process may come across as contradictory for a framework aimed in improving student engagement. However, we argue that this process should be seen as an engagement milestone. It provides the students an opportunity to plan for the production of a work that is likely to meet required standards, reducing the chances of disengagement and failure at a later stage. To the best of our knowledge, we are the first to embed such a vetting process within the implementation of FYPs in the Computer Science subject area at UK universities.

\section{Assessment Breakdown}

The developed framework uses Experimental Learning Theory [18] to ensure constructive alignment at both the module and programme-level. The framework also provides an assessment that corresponds to the learning goals, which results in the construction of thinking, knowledge skills and subjectbased practical skills. Instructional scaffolding has also been incorporated in the framework for students via Assessment for Learning [27] techniques which uses a range of diagnostic, information, continuous formative and summative assessments as tools and milestones throughout the year-long project.

The summative assessments leading to marks for the project are divided over two assessment components and culminates the multiple milestones from Figure 1. The breakdown of the components is given in Table I. In our particular case, students needed to achieve a threshold mark of $40 \%$ for each component to pass the project. It should be noted that some values described in this section, such as, the number of weeks for lectures, number of engagements with supervisors, checkpoints etc. are tied to our implementation of the framework. These can be easily replaced with other values or components to meet institutional and discipline requirements.

\section{Framework in Practice}

The practical implementation of this framework also involved the following:

1) Formal Lectures: The formal lecture and workshops were presented through a series of 2 hours sessions which was class-room based and instructor led. The topics that were covered during these sessions included - introduction to individual project, research methods, literature reviews, development in practice, personal development planning, use of electronic resources, plagiarism \& referencing, legal \& ethical issues, and preparing for assessments.

2) Selection Process of Assessors: The assessment of the project is conducted by the supervisor and an independent assessor who is also referred to as the second marker. The supervisor is often not allocated but is decided mutually between the supervisor and the student based on commonality of interest or based on prior interaction. As the supervisor is expected to intricately support the student and their project, the supervisor is likely to become personally invested in the project work. Although this puts the supervisor in the best position to assess the work produced, it raises the possibility of biased judgement.

For this reason, the allocation of the second marker is done by the project team independently and is not chosen by either the supervisor or the student. This ensures objective and transparent assessment of the work produced. Particular care has also be taken to ensure that the supervisor and second assessors pairing avoid cases where the same pair of academics a fulfilling the roles for each others students as that may lead to yet another bias. The second marker is only involved in the assessment of the work and does not have any interaction with the student's project in terms of guidance or support.

3) Training Workshops: Training workshops for academics and students were organized with the purpose of providing guidance about the project process, formative and summative assessments, sharing of templates and using the SharePoint project sites. The main advantage of these workshops is to manage expectations, raised awareness of the requirements and encourage consistency in the way supervision and assessment is carried out. In our case, we also supported students with specific workshops that provided them with training on how to use SharePoint to build project sites.

4) Assessment Process: As FYPs are unstructured in their nature and the projects vary in the topics that they address, the assessment process needs to be able to cater for the creative variation while attempting to keep consistency. As such, the same marking scheme, guidance and training has been provided to each assessor to support the consistency and benchmarking. We also advise the use of blind marking and review between the supervisor and the second marker. However, this results in the possibility of a discrepancy in marking. In cases where the marks vary by more than 10 points or where the assessors puts the students work in different classification (pass / fail), a third assessor is engaged to provide an independent mark based on the feedback and the marks provided by the supervisor and the second marker.

\section{EMPIRICAL VALIDATION}

This empirical validation investigates the effectiveness and impact of the framework on the performance of students conducting their final year projects within a Computer Science department at a UK university. In total we consider the performance of 625 students of a period of 5 year for the 
academic year 2012/13 - 2016/17. The following research questions are addressed within this section:

$R Q 1$ - What was the performance trends of student cohorts undertaking their final year projects?

$R Q 2$ - Given the pre- and post- framework introduction, what is the performance variance of non-project and project modules?

In order to assess the effectiveness of the enhanced framework, we analyse student performance in terms of pass rate $(P R)$ as the percentage of engaged students who have passed the module and average marks $(A M)$ of students to be critical indicators. We also consider engagement rate $(E R)$ as the percentage of engaged students to be another crucial measure for the success of the framework.

For a module $m$, let, $m_{S}=\left\{s_{1} \ldots s_{x}\right\}$, be the set of enrolled students, $m_{S E}=\left\{s e_{1} \ldots s e_{y}\right\}$ be the set of students who attempt all required assessments (i.e. engaged with the module), $m_{S P}=\left\{s p_{1} \ldots s p_{z}\right\}$ be the set of students who are awarded a pass grade, and, $\operatorname{marks}\left(s e_{i}\right)$ is the marks received by student $s e_{i}$. Therefore, $m_{S P} \subset m_{S E} \subset m_{S}$. We define the following measurement indicators for any module $m$ :

$$
\begin{aligned}
& P R=\frac{\left|m_{S P}\right|}{\left|m_{S E}\right|} * 100 \\
& E R=\frac{\left|m_{S E}\right|}{\left|m_{S}\right|} * 100 \\
& A M=\frac{\sum_{i=1}^{y} \operatorname{marks}_{\left(s e_{i}\right)}}{\left|m_{S E}\right|}
\end{aligned}
$$

(Pass Rate)

(Engagement Rate)

(Average mark)

\section{A. Project Performance Trends}

In this section we present the performance trends for the final year project module for the past five years and address research question $R Q 1$.

TABLE II

COHORT Performance Data ( $m=$ FYP Module $)$

\begin{tabular}{c|c|c|c|c|c|c|c}
\multicolumn{8}{c}{ COHORT PERFORMANCE DATA $(m=$ FYP MODULE $)$} \\
\hline & Year & $m_{S}$ & $m_{S E}$ & $m_{S P}$ & $E R$ & $P R$ & $A M$ \\
\hline Pre & $12 / 13$ & 109 & 90 & 74 & 83 & 82 & 56 \\
framework & $13 / 14$ & 167 & 110 & 98 & 66 & 89 & 60 \\
& $14 / 15$ & 135 & 70 & 55 & 52 & 79 & 57 \\
\hline Post & $15 / 16$ & 122 & 102 & 87 & 84 & 85 & $\mathbf{6 5}$ \\
framework & $16 / 17$ & 92 & 80 & 73 & $\mathbf{8 7}$ & $\mathbf{9 1}$ & 62 \\
\hline Total: & - & 625 & 452 & 387 & - & - & - \\
Average: & - & 125 & 90 & 77 & 74 & 85 & 60 \\
\hline
\end{tabular}

Table II presents the performance data for students undertaking their final year project in the subject area of Computing during the academic years 2012/13 - 2016/17. Column 3 of the table shows the number of students enrolled on the FYP module, Column 4 shows the number of students who attempted the assessments of the module, Column 5 shows the number of students who achieve a pass grade or higher in the module. Column 6, 7, and 8 provides the $E R, P R$ and $A M$ values for the FYP module for the corresponding academic year. The total number of students considered within the study is 625 .

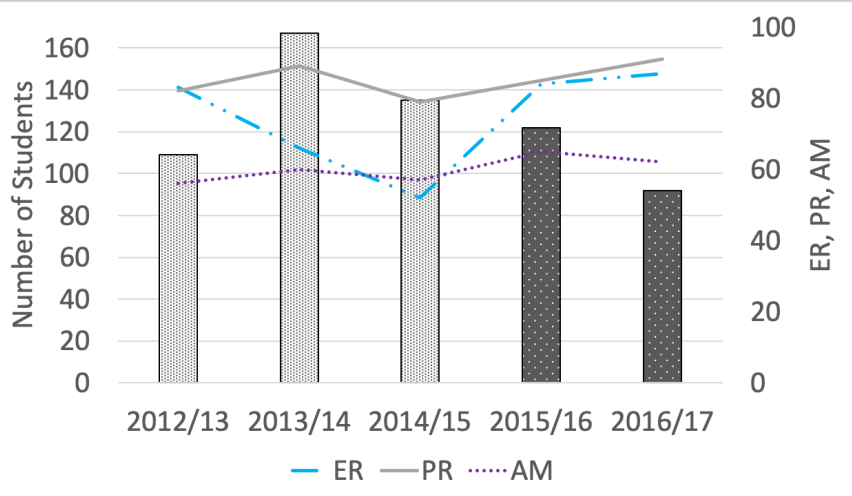

Fig. 3. Student Performance in Project Module

The primary way to determine the effectiveness of the enhanced framework is to study its impact on the pass rate $(P R)$. It can be seen from Figure 3 that there is an increase in pass rate with the adoption of the framework in 2015/16, where the rate increased by $6 \%$ to $85 \%$ as opposed to the previous year. The pass rate actually continues to rise sharply to $91 \%$ (to its highest ever) in 2016/17 during the second year of the framework implementation. We therefore see a steady improvement in the pass rate since the introduction of the framework. This rise in the pass rate in Implementation 2 is due to the introduction of the Final Proposal Vetting process, which allowed for the identification and withdrawal of students with inadequate project plan/proposal early in the module cycle as discussed in Section IV-B. Another interesting observation is the significant low pass rate in 2014/15. The reason is possibly down to the limited usage of milestones and the overall module being judged using a single assessment component, which is the end of the year project report. One of the principal contributions of the framework is the combination of regular assessment milestones both formative and summative, which serve as building blocks for the entire project.

Another metric to measure the effectiveness of the enhanced framework is the student engagement rate $(E R)$. This is determined by the number of students who attempted the assessment components given the total number of students who were enrolled on to the project module. According to Figure 3, the engagement rate of students have been falling sharply from $83 \%$ in $2012 / 13$ to $52 \%$ in $2014 / 15$. When the framework was first introduced in 2015/16 to address this issue, the student engagement shot up to $84 \%$, higher than the prior 3 years. In 2016/17 during the second year of the framework's implementation, the engagement grew further to its highest level ever at $87 \%$. This is also far higher than the $80 \%$ degree completion rates for 2016/17 reported by the Higher Education Statistics Agency (HESA) [1] across all Higher Education institutions in the UK.

The final metric for assessing the framework is the average mark $(A M)$, which is based on the average of all marks given the total number of students who attempted the assessment components. Figure 3 shows that the average mark remained within the 56 to 60 range pre-framework implementation. 
The average mark increased to 65 after the framework was introduced in 2015/16. However, this went down by a few points in the following year, still an overall improvement.

Therefore, as an answer to the research question $R Q 1$, we find that the pass rates were fluctuating for the three years prior to the introduction of the framework and the lowest of the pass rates was observed in 2014/15 with only $79 \%$ students passing the module. In term of student engagement, we find a steady decline from $2012 / 13$ to $2014 / 15$ where the engagement rate drops from $83 \%$ to an abysmal $52 \%$. Finally, the combined average marks of students for the three years prior to the introduction of the framework was 58. On the other hand, we see that the introduction of the framework shows improved results in all three metrics. The pass rate shows a steady growth in both years, with the highest of $91 \%$ in 2016/17, similarly the student engagement rate has also increased in both years hitting the highest of $87 \%$ in $2016 / 17$. Finally, the combined average marks for both years where the framework was implemented stands at 64 , giving an average improvement of six marks compared to the prior three years.

\section{B. Cohort Performance Analysis}

The results in Figure 3 are promising. However, they do not consider the fact that the cohorts for each academic year were different, and could lead to subject bias. Although inclusion of multiple cohorts from pre- and post- framework introduction are considered, which alleviates this phenomenon. However, it can still be argued that the observed improvements could have been due to stronger cohorts over the last two years. Therefore, we will address research question $R Q 2$, by comparing the performance of the three cohorts (one preframework, one post-framework introduction and one postframework enhancement) in all their academic modules with the results of the project module. If there is an improvement found in the marks for the project module compared to the non-project modules, then this can be credited to the framework and not the cohort.

We have classified students that started their three-year BSc programme in 2012/13 as Cohort 1, while students who started in 2013/14 have been classified as Cohort 2, and students who started in 2014/15 have been classified as Cohort 3. Students in Cohort 1 did not conduct their project under the framework, while students in Cohort 2 did their projects during Implementation 1 and students in Cohort 3 during Implementation 2. Based on this we have compared the project performance of the cohorts with other modules that they undertook during those periods, the results of which can be seen in Table III.

TABLE III

NON-PROJECT MODULE VS PROJECT MODULE PERFORMANCE

\begin{tabular}{c|c|c|c}
\hline Cohort & $\begin{array}{c}\text { Non-project Module } \\
\text { Average Mark }\end{array}$ & $\begin{array}{c}\text { Project Module } \\
\text { Average Mark }\end{array}$ & $\begin{array}{c}\text { Project Module } \\
\text { Pass Rate }(\%)\end{array}$ \\
\hline 1 & 55 & 57 & 79 \\
2 & 51 & 65 & 85 \\
3 & 55 & 62 & 91 \\
\hline
\end{tabular}

Three of the cohorts had very similar average marks $(55,51$, 55 ) for their modules (excluding the project) during years 1 to
3, which indicates that all cohorts were very similar in terms of their academic performance. The similarity in the performance of the cohorts makes it possible to make an accurate performance comparison of the proposed project framework with the previous project module. It can be seen in Table III that the project pass rate for Cohort 2 and Cohort 3 was $85 \%$ and $91 \%$ as opposed to the $79 \%$ for Cohort 1 , which is a meaningful improvement. In addition to this, the average mark for the projects conducted by Cohort 2 and Cohort 3 were 65 and 62, while Cohort 1 was 57. These results strongly indicate that the framework directly impacted the performance of students on the project module.

In response to $R Q 2$, it can be said that the performance on non-project based module was very similar for cohorts from pre-framework introduction and post-framework introduction. However, the project pass rate showed a $6 \%$ improvement for Cohort 2, whereas the average mark also increased. Both of these strongly indicate that the positive change was a direct consequence of the introduction of the framework.

In terms of assessing the impact of the changes introduced during the second implementation of the framework, we can see that the project pass rate showed a further $6 \%$ improvement for Cohort 3. This is due to the number of students who benefited from the final proposal feedback, as 15 students needed a second attempt to ensure that their final proposals were technically sound for a final year project. These are students who may have potentially failed the project if the final proposal assessment was not introduced in 2016, as it gave these students an opportunity to rectify their proposal based on feedback received from the assessing panel. Therefore the number of students that passed in the 2nd attempt account for the further increase in the pass rate in 2016/17. We did not withdraw any students, as the students who needed a 2nd attempt for the proposal managed to address the changes from the panel and met the required standards. The final proposal milestone helped supported engagement for students who may have otherwise disengaged later due to lack of appreciation of the required standards, insufficient resources or incorrect scoping.

\section{Threats to Validity}

The threats to the validity of the results presented here are primarily from the subject bias that may be introduced because of multiple cohorts. This is always a possibility for longitudinal studies of this nature that compare results from different cohorts. We alleviate some of this concern by introducing secondary results (non-project modules) as a baseline.

Another threat is the assessors for the project assessment, as they may have changed for the various cohorts. However, to address this, we conducted a series of training sessions to ensure consistency in marking and understanding of the project process. In addition to this, we also performed third marking when there was a marking discrepancy exceeding a certain threshold. 
The final threat arises from the institutional context and its impact on the success and effectiveness of the framework, which may indicate inability to generalize the framework to other institutions and departments. During the implementation we minimized external factors as much as possible and are looking to do extended studies in alternate environments to assess generalizability.

\section{CONCLUSiOnS AND Future WORK}

In this paper, we address the challenge of student engagement and performance by extending a teaching \& learning framework for computing final year projects that was implemented within a Computer Science department at a UK university. Empirical validation shows that the pass rate improves by $12 \%$ and engagement rate improves by a staggering $35 \%$ following implementation of the framework. Feedback from colleagues, students and external reviewers were overwhelmingly positive and lead to the module team receiving an institutional award for Teaching Innovation. Going forward, we want to implement the framework at Computing departments at other universities across the UK and even across other disciplines to show generalizability.

\section{ACKNOWLEDGEMENTS}

This work was supported by the ..., which is supported by the ...

\section{REFERENCES}

[1] H. E. S. Agency. Non-continuation summary: Uk performance indicators 2016/17. http://bit.ly/2CugLxY, 2018. Accessed: 2018-11-25.

[2] A. Author, A. Author, and A. Author. The tile is annonymised to support the blind review process of the conference. In The venue is annonymised to support the blind review process of the conference., 2019.

[3] S. Bloxham and P. Boyd. Developing Effective Assessment In Higher Education: A Practical Guide: A Practical Guide. McGraw-Hill Education (UK), 2007.

[4] S. D. Brookfield. Becoming a critically reflective teacher. John Wiley \& Sons, 2017

[5] C. Bryson and L. Hand. The role of engagement in inspiring teaching and learning. Innovations in education and teaching international, 44(4):349-362, 2007.

[6] L. Cohen, L. Manion, and K. Morrison. Research methods in education, 2007.

[7] F. D. Davis, R. P. Bagozzi, and P. R. Warshaw. User acceptance of computer technology: a comparison of two theoretical models. Management science, 35(8):982-1003, 1989.

[8] S. Freeman, S. L. Eddy, M. McDonough, M. K. Smith, N. Okoroafor, H. Jordt, and M. P. Wenderoth. Active learning increases student performance in science, engineering, and mathematics. Proceedings of the National Academy of Sciences, 111(23):8410-8415, 2014.

[9] L. Gambin, R. Beaven, T. Hogarth, M. May-Gillings, and K. Long. Methodological issues in estimating the value added of further education, higher education and skills: A review of relevant literature. 2014.

[10] N. A. Gordon. Issues in retention and attainment in computer science. York: Higher Education Academy, 2016.

[11] M. Healey, L. Lannin, A. Stibbe, and J. Derounian. Developing and enhancing undergraduate final-year projects and dissertations. Higher Education Academy Gloucestershire, 2013.

[12] S. I. Ismail, R. Abdullah, S. A. C. Kar, N. Fadzal, H. Husni, and H. M. Omar. Online project evaluation and supervision system (opens) for final year project proposal development process. In Student Conference on Research and Development, pages 210-214. IEEE, 2017.

[13] M. Kangas, P. Siklander, J. Randolph, and H. Ruokamo. Teachers' engagement and students' satisfaction with a playful learning environment. Teaching and Teacher Education, 63:274-284, 2017.
[14] P. Khamaruddin, A. Sauki, N. H. O. Kadri, A. N. C. A. Rahim, and A. Kadri. Technology acceptance model analysis on students' behavioral intention of using moodle for fyp. In World Engineering Education Forum, pages 724-727. IEEE, 2017.

[15] J. LeBihan, C. Hughes, and C. A. Taylor. The teaching excellence framework: Quality, equality and student engagement in english higher education. In Contexts for Diversity and Gender Identities in Higher Education: International Perspectives on Equity and Inclusion, pages 141-154. Emerald Publishing Limited, 2018.

[16] M. Malik, R. Khusainov, S. Zhou, and V. Adamos. A two year case study: Technology assisted project supervision (tapas). engineering education, 4(2):76-83, 2009.

[17] A. J. Martin. The student motivation scale: A tool for measuring and enhancing motivation. Journal of Psychologists and Counsellors in Schools, 11:1-20, 2001.

[18] I. McGill and L. Beaty. Action Learning: a guide for professional, management \& educational development. Psychology Press, 2001.

[19] I. Ortiz Marcos, Á. Uruburu Colsa, S. Ortiz Marcos, and R. Caro Carretero. Final year project: students and instructors perceptions as a competence-strengthening tool for engineering students. International Journal of Engineering Education, 28(1):83-91, 2012.

[20] J. Pickering. How to start using technology in your teaching. http: //bit.ly/2PVuGQN, 2015. Accessed: 2018-11-25.

[21] K. A. Renninger and J. E. Bachrach. Studying triggers for interest and engagement using observational methods. Educational Psychologist, 50(1):58-69, 2015.

[22] R. Säljö. Digital tools and challenges to institutional traditions of learning: technologies, social memory and the performative nature of learning. Journal of computer assisted learning, 26(1):53-64, 2010.

[23] R. K. Sawyer. The Cambridge handbook of the learning sciences. Cambridge University Press, 2005.

[24] S. Shay. The assessment of undergraduate final year projects: A study of academic professional judgment. $\mathrm{PhD}$ thesis, University of Cape Town, 2003.

[25] P. Siklander, M. Kangas, S. Ruhalahti, and S. Korva. Exploring triggers for arousing interest in the online learning. In International Technology, Education and Development Conference, pages 9081-9089, 2017.

[26] H. Singh. Building effective blended learning programs. Educational Technology-Saddle Brook, 43(6):51-54, 2003.

[27] TLR. Effective learning and teaching in uk higher education. https: //goo.gl/6C1XUj, 2015. Accessed: 2018-11-25. 